\title{
The mechanical property prediction of fibre-reinforced composite materials based on a new generating random fibre distributions method
}

\author{
Heyuan Huang ${ }^{1, a^{*}}$, Meiying Zhao ${ }^{1, b}$, ,Yonghui Dai ${ }^{2, c}$, Wenzhi Wang ${ }^{1, d}$ \\ ${ }^{1}$ School of Aeronautics, Northwestern Polytechnical University, Xi'an Shaanxi, 710072, China \\ ${ }^{2}$ Shanghai Aircraft Customer Service Co.,Ltd. Shanghai, 200241, China \\ anpuhhy@nwpu.mail.edu,cn, bzhaomeiying@nwpu.edu.cn, cdyhxgd@126.com \\ 'Wangwenzhi@nwpu.edu.cn,
}

\begin{abstract}
Keywords: represent volume element (RVE), fibre distributions, image processing, statistical description, composite, elastic properties prediction.

Abstract: This paper focuses on the issues of the fibre-reinforced composite material microstructure and presents a new numerical method to generate random fibre distributions and predict the macroscopic mechanical properties of composite materials based on the represent volume element (RVE). The method, named the random sequence generation algorithm (RSGA), obtains fibre radius distributions and positional relationships by image processing to generate a statistical equivalence RVE effectively. On this basis, the composite material microstructure finite element model is established to predict its macroscopic elastic properties by Python language in ABAQUS. Comparative analysis shows the algorithm is in good agreement with experimental results and has the statistical equivalence herein. The research of this paper on the algorithm for composite materials of fibre distributions provides a useful alternative to generate random numerical models that can be used in micromechanical analysis of composite materials.
\end{abstract}

\section{Introduction}

In recent years, the rapid development of composite materials leads to a wide range of application on many fields, especially in the aerospace industry for their excellent performance. According to structure characteristics, composite materials can be divided into three kinds: sandwich composite materials, fines composite materials and fibre-reinforced composite materials ${ }^{[1]}$. In the fibre-reinforced composite materials, fibre is the main part to carry most of the load. Therefore, fibre volume fraction and fibre distribution morphology have crucial influence on the strength and stiffness properties of composite materials.

Current research methods of composite materials properties include experimental study and numerical calculation. The experimental way is able to give a complete property description for a certain material while it requires a considerable amount of labor and financial resources. In the numerical calculation terms, finite element analysis with a representative volume element (RVE) is a highly attractive approach, because of its clear thinking and simple algorithm ${ }^{[2,3]}$.

Sun predicted the elastic modulus and Poisson's ratio of the particle reinforced composites by the fibre randomly distributed representative volume element and learned that the result of this model simulated better than the fibres regularly distributed model compared with the experimental values ${ }^{[4]}$. Hojo.M studied the local fibre distribution effect on the micro-interface normal stress of unidirectional carbon fibre epoxy laminates under transverse tensile loading and the results showed that the interface normal stress absolute value of fibre irregular distribution increased rapidly when the distance between fibres was less than $0.5 \mu \mathrm{m}^{[5]}$. Vaughan and McCarthy presented the nearest neighbour algorithm based on test data and its local shape distribution function was equivalent to the fibre distribution of real structure ${ }^{[6]}$. AR Melro used the hard-core model to generate fibre randomly distribution model which the fibre volume fractions could be up to $65 \%{ }^{[7]}$. Matsuda draw a conclusion that fibre distribution type in the RVE did not affect the macroscopic properties of laminated plate but had a huge impact on the microscopic stress distribution ${ }^{[8]}$. Romanowicz found that fibe distribution types not only had a major influence on the matrix crack initiation but also could lead to stress concentration and strain localization ${ }^{[9]}$. Lei Yang proposed the random sequential 
expansion method which was equivalent to the completely random type (CSR) and could quickly generate a high volume fraction $\mathrm{RVE}^{[10]}$.

In composite micro-structures, fibre distribution is so discrete and randomized that the traditional periodic representative volume element cannot describe the whole mechanical properties for composite materials. Therefore, in this paper, a new algorithm based on the hard-core model is presented to describe the random distribution characteristics of fibre and applied to establish a representative volume element model of composite microstructure to complete the prediction of the macroscopic mechanical properties. The predicted effective properties are compared with experimental results and verified by four statistical functions.

\section{Microstructure feature extraction of composite materials}

\section{Image Processing}

In the Matlab, every image corresponds to a certain matrix, especially, for a binary image, the black and white elements are expressed by 0 or 1 respectively ${ }^{[11]}$. For a composite microstructure SEM scanning image, geometrical characteristics and microstructure features of the target region are obtained through binary image processing.

Figure.1-a is the microstructure SEM scanning image of T300/Epoxy composite which the fibre volume content is $57 \%^{[12]}$. First of all, the convert true color picture is transferred to grayscale image by rgb2gray (I) function in Matlab. Then, the original grayscale image gradation distribution area is centralized for the purpose of increasing the contrast and making clear image details. Finishing above steps, the image is processed into two values by the way of selecting an appropriate threshold with the $\operatorname{im} 2 b w(I, a)$ function in Matlab. In this paper, the threshold is solved by the iterative method and the final value is $133^{[13]}$. The last step is the binary image morphological processing which includes four arithmetic operators: expansion, corrosion, open and close. The result obtained by image processing is shown in Figure.1-b.

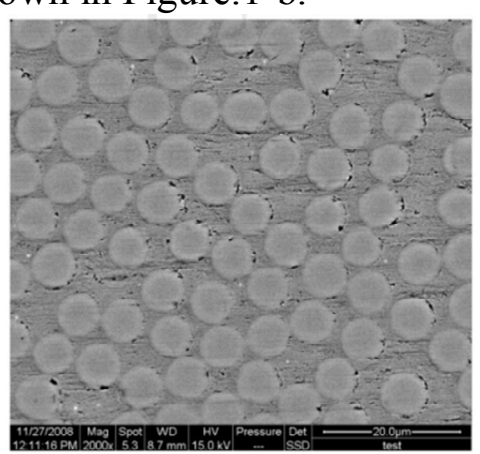

a)The real micro strcuture of T300/Epoxy

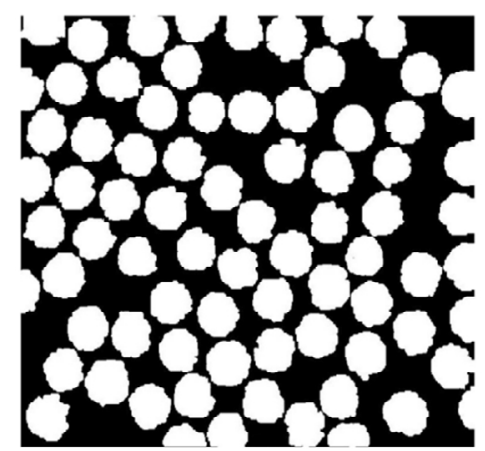

b)The result of binary image process

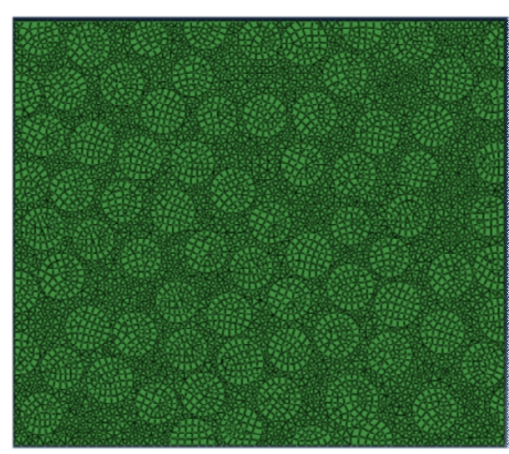

c)The finite element model

Figure.1 Microstructure of T300/Epoxy

\section{Finite element model}

Geometric characteristics of composite microstructure consist of two parts: fibre area and fibre centroid. According to the data obtained from the pixel distribution reading result in Figure.1-b, the finite element model is automatically generated by the Python language in ABAQUS as shown in Figure.1-c. It can be seen from the comparison of Figure.1-a to 1-c that above image processing method can accurately get microscopic distribution of the fibres, which is the foundation to establish a reasonable RVE.

\section{The random sequence generation algorithm (RSGA)}

For composite materials with high fibre volume fraction, fibres were frequently generated by the random sequential expansion (RSE) method and the nearest neighbour algorithm (NNA) in RVE. The 
former ${ }^{[10]}$ can overcome the limitation of hard-core model ${ }^{[14]}$. However, when the fibre volume content was reached, the fibre may not be extended to the corner of RVE and it was easy to appear in the middle or somewhere centralized. The $\mathrm{NAA}^{[16]}$ used the nearest distance distribution function to define the distance of different fibres in RVE. Its advantage included short execution time, basically consistent with the statistical distribution and reasonable simulating of the fibres microscopic characteristics. However, both the distribution of the fibre radius and the nearest distance of the RVE generated by the NAA were less than experimental results for the reason that the criterion condition which prevented the nearest fibres from overlapping was so easy to content and leaded to the smaller diameter.

In the paper, a fast fibre distribution generation method equivalent to statistical data is proposed and named the random sequence generation algorithm(RSGA). The algorithm generates fibre distance and fibre radius to model the micro structure with statistical data through analyzing 2D material cross-sectional images. Then, an equivalent model is re-established based on both classic hard-core hypothesis model ${ }^{[14]}$ and the SEM images which represent actual fibre distribution.

The steps of random sequence generation algorithm are as follows:

Step 1: The first fibre is generated randomly in the target area and taken as the so called "current fibre"; then, its closest distance with other new fibres is given in accordance with the nearest distance distribution function which is shown in Figure.2-a.

Step 2: Generation of new fibre surrounding the "current fibre". The position of the new fibre is determined by the nearest distance and its angle with the "current fibre" which is shown in Figure.2-b.

Step 3: If the nearest distance between the new fibre and the "current fibre" is less than the nearest distance, then the new one is regenerated. To prevent the nearest distance of new fibres becoming larger and larger, probability selection is added for each inspection failure. When the random probability value $P$ is less than $P_{0}$, even though the new generation fibre's nearest distance does not meet the above requirements, the fibre is still generated. According to experience, $P_{0}$ is taken as 0.15 .

Step 4: Verifying the distance between new generations of fibres with others. The generation should be given up if the distance is less than the nearest distance with other labeled fibres or new fibres. For example, the 4th fibre is regenerated if the distance between the 4th and the 2nd fibre is less than the nearest distance of the 2nd fibre, as shown in Figure.2-c.

Step 5: Updating the fibre nearest distance distribution, the radius distribution and the generated fibres. Probability of fibre nearest distance updating formula (1) is as follows:

$$
P_{i}=\frac{P_{i}^{0} N-N_{i}}{N-\sum N_{i}}
$$

Which $P_{i}$ represents the updated nearest distance generation probability of the area of $i$. $P_{i}^{0}$ represents the nearest distance generation probability of the initial area $i$. N represents the total predicted number of fibres. $N_{i}$ represents the total number of generated fibres in the area $i$.

Step 6: End the cycle if there is no new fibre generation surrounding the target fibre after a certain number of attempts.

Step 7: The fibre is selected as the "current fibre" to generate the new fibre one by one until meeting the target fibre volume fraction in the RVE or reaching the maximum number of cycles. Then, stop generating new fibres and output the results.

Figure. 3 is the flowchart of random sequence generation algorithm which $f(i)$ represents a random fibre, $d_{n e w}$ is the nearest distance of the new fibre and $d_{c f}$ is the nearest distance of the "current fibre". 


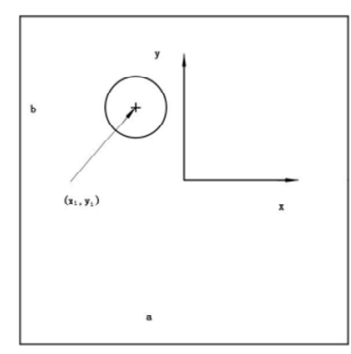

a)Generate the first fiber

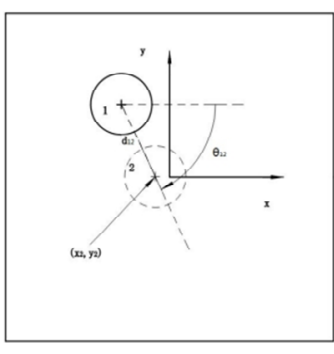

b)Generate the next fiber

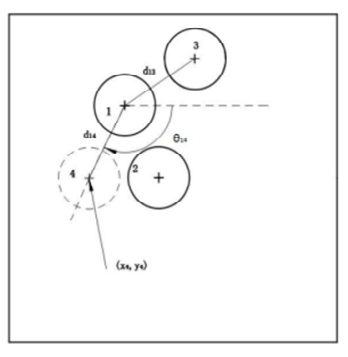

c)Inspect the new fiber

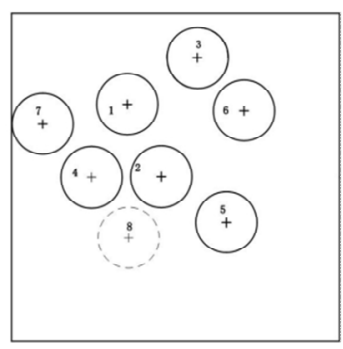

d)The generated fibers

Figure. 2 The method of advanced NNA

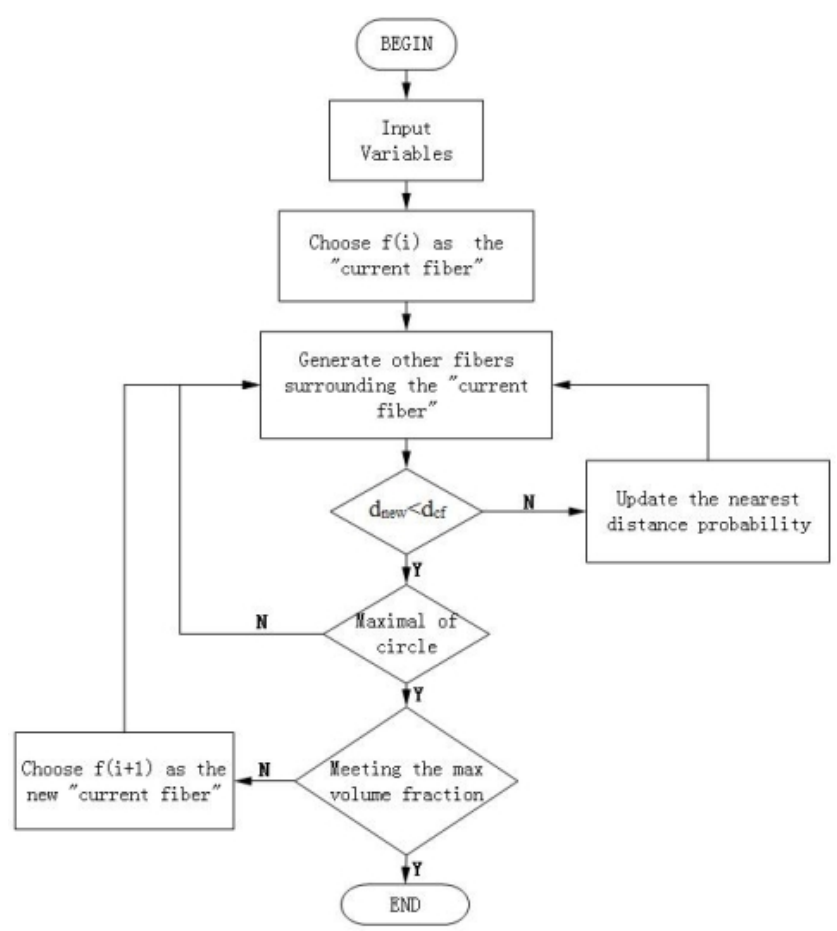

Figure.3 Flowchart of random sequence generation algorithm

\section{Statistical characterization analysis}

In the paper, the random fibres distribution state of different RVE generating methods are evaluated by four kinds of statistical functions ${ }^{[15]}$ from various aspects of statistical characterization.

\section{Nearest neighbour fibre distances distribution function}

The nearest distance fibre distances distribution is an important feature of composite microstructure models and the initial damage laminates generally occurs where fibres are much close to each other ${ }^{[16]}$. PDF (Probability Density Function) is defined by the minimum distance value of the target fibre with its neighbour fibre and it can provide information on the short-range interactions between fibres. The fibre aggregation phenomenon takes place if the PDF function shows a spike and a followed steep drop in a specific value place.

Different algorithms including RES and NAA are used to generate 20 sets of RVE and their nearest fibre distance distributions are shown in Figure.4. It can be seen that both results of experiment and the RSGA basically obey the normal distribution. Otherwise, the outcomes deviate significantly from experimental values and present as a uniform distribution for the RVE models achieved by the RSE. Comparison illuminates that the fibre distribution depends on the fibre generation method. What's more, for the random sequence generation algorithm, the error with the experimental result is small enough to meet the requirements of statistical equivalence on the nearest distance distribution. 


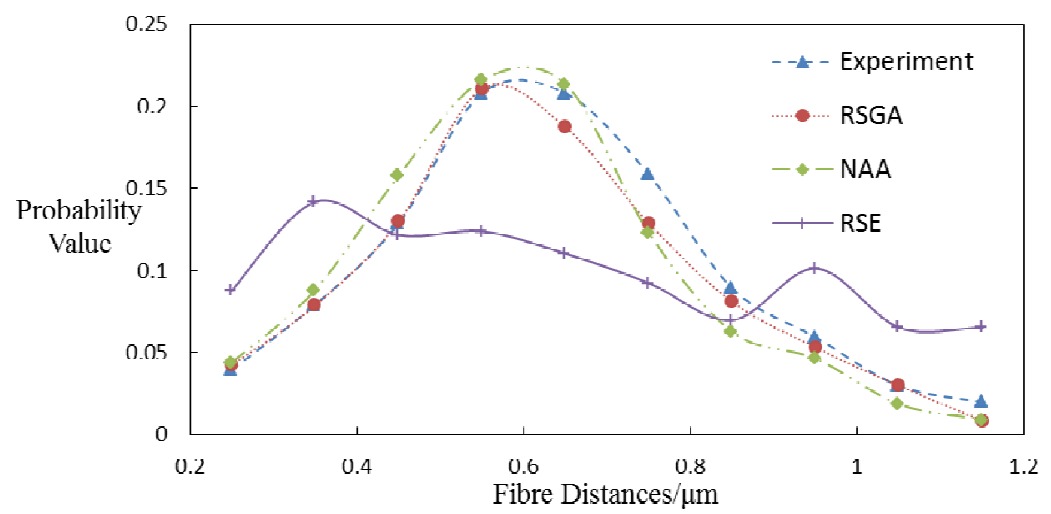

Figure.4 The nearest fibre distance distribution

\section{Nearest neighbor fibre orientation distribution function}

In the paper, the nearest neighbor orientation of the target fibre is described with the cumulative distribution function (CDF). The CDF is specifically defined as the turning angle that one fibre rotates to its nearest fibre connecting center line along the horizontal axis in a clockwise direction. The CDF is normalized with respect to the total number of fibres and represents a straight diagonal line when the fibre distribute completely random in the RVE, meaning that a given orientation has the same probability of occurring as any other orientation. If the fibre distribution in the space is very close in a certain direction, such as the periodic distribution, the CDF curve should deviate significantly from the diagonal line and present a step shape. Figure. 5 has compared the CDF curves between experiment results and the RSGA which indicates the fibre orientation distribution is an entirely random nature of statistical equivalence.

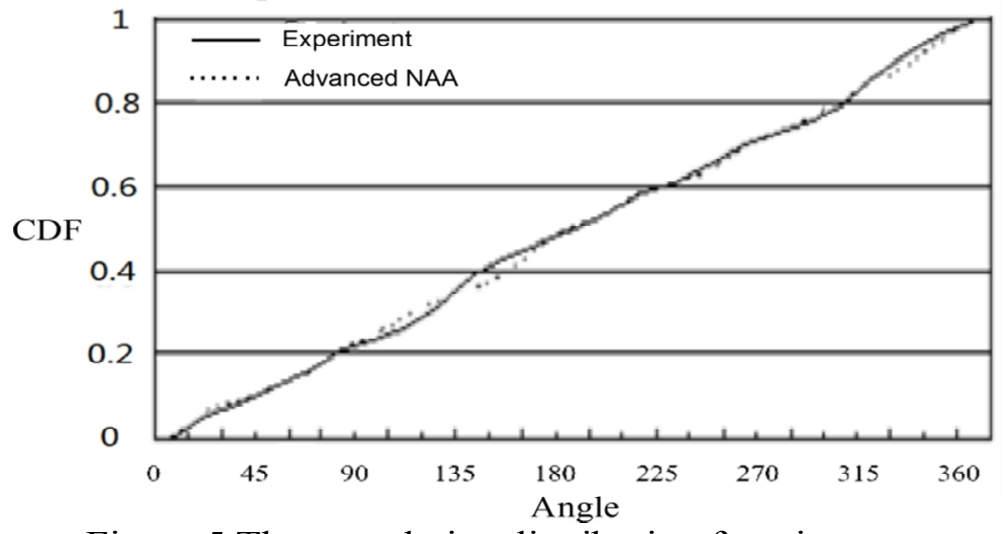

Figure.5 The cumulative distribution function curves

\section{Ripley's K function}

Ripley's K function, also known as the second-order intensity function, is mainly used to describe the spatial distribution of information ${ }^{[16]}$. Ripley's $\mathrm{K}$ function $K(h)$ is calculated in formula (2):

$$
K(h)=\frac{A}{N^{2}} \sum_{i} \sum_{j \neq i} \frac{I\left(d_{i j} \leq h\right)}{w(i, h)}
$$

Where $A$ is the area of the window, $N$ is the total number of fibres, $d_{i j}$ is the distance between points $i$ with $j, I()$ is an indicator function having the value 1 if the condition between brackets holds true and the value 0 if the condition is false. $w(i, h)$ is a weight function having the value 1 if the circle with center at point $i$ and passing by point $\mathrm{j}$ is completely inside the window. Otherwise, $w(i, j)$ is the ratio of the circumference contained within the window to the whole circumference of the circle.

In order to compare different methods of $K$ function more clearly, the formula (2) can be expressed by the representative of the formula (3): 


$$
L(h)=\sqrt{\frac{K(h)}{\pi}}-h
$$

By the formula (3), the possion distribution constant corresponding to $L(h)$ is 0 . For different distances $h$, the positive peaks of the $L(h)$ curves represent that the fibre is aggregated and the negative peaks represent the regular distribution of the fibre. Curves of different methods are shown in Figure.6, where the average radius of fibre is $3.05 \mu \mathrm{m}$.
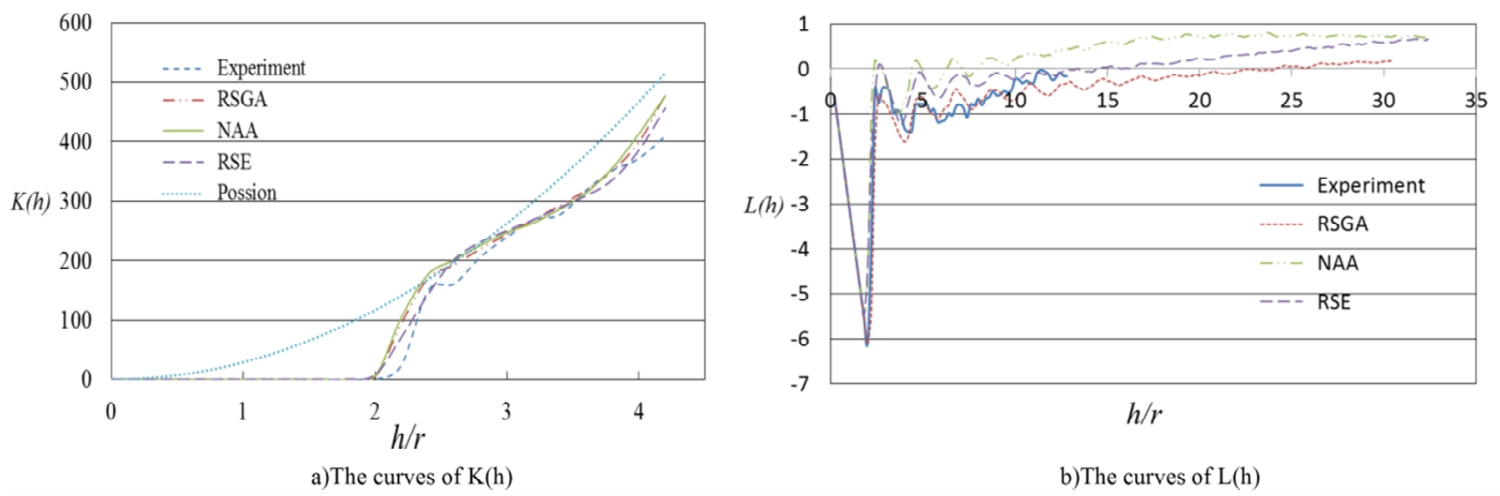

Figure.6 Curves of different Ripley's K functions

It can be seen from the Figure. 6 that the RSGA has the minimum error with experimental values than other methods which are significantly deviates from the experimental values. Wherein, the curves obtained by NNA and RSE agree with the possion distribution. In summary, the methods based on the complete random distribution are not suitable for simulating the real fibre distribution and the RSGA is relatively closer to the type of real fibre distribution and appropriate to simulate the real distribution of fibres.

\section{Pair distribution function}

Pair distribution function is used to describe the relationship between average fibre density and distance of the fibres inside RVE. Pair distribution function $G(h)$ is defined as the formula(4): the probability of finding an additional point within an annular region of inner radius $r$ and outer radius $r$ $+d r$ respectively. Therefore, for the statistical distribution of the fibres, $G(h)$ should be increased gradually with the increase of $h$ and gradually close to $1^{[18]}$.

$$
G(h)=\frac{1}{2 \pi N_{a} h \cdot d h} \cdot \frac{1}{N} \sum_{i=1}^{N} n_{i}(h)=\frac{1}{2 \pi h} \cdot \frac{d K(h)}{d h}
$$

Where the $d K(h)$ is the average number of fibres between the inner diameter of $h$ and the outer diameter of the $h+d h . N_{a}$ is the number of fibres per unit area ${ }^{[19]}, n_{i}$ is the fibre center number in the ring. Figure.7 shows a large spike at about $2.2 r$ of the pair distribution function, which is corresponding to the spikes of the nearest neighbour fibre distances distribution function. The radial distribution function curve tends to be stable at 1 when the value of $h$ increases to large enough to meet the needed minimum size of the composite mechanical properties analysis. Figure.7 shows $G(h)$ obtained by the RSE is less than the experimental value and quickly converge to 1 in the interval of 5 to 10 . The $G(h)$ of the NAA and the RSGA are consistent with the experimental measured data relatively and also tends to 1 in the progressive when the value of $h / r$ is large than 15 . 


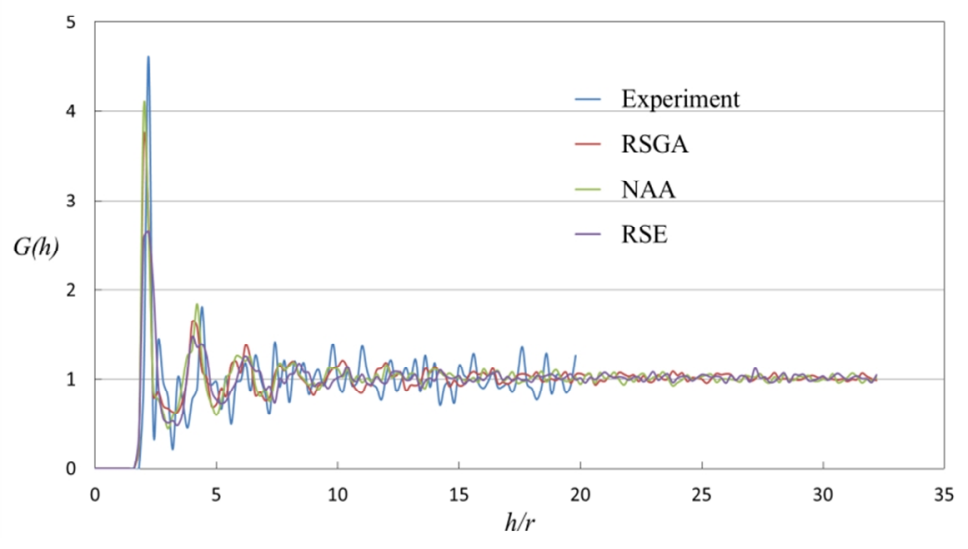

Figure.7 Curves of the pair distribution function

\section{Mechanical property prediction of the composite material}

In this paper, the macroscopic elastic property of T300/BMP-316 is predicted by the finite element method based on micromechanics. By applying appropriate periodic boundary conditions, the RVE model data of stress-strain response under different uniaxial load can be achieved. Then, the elastic properties of the composite materials are calculated according to the homogenization theory and compared with the experimental data.

\section{The finite element model of a single cell}

Twenty sets of microscopic finite element cell model are established for calculation and the length of the RVE side is chosen as 50 times as fibre average radius ${ }^{[20]}$, each RVE model contains total 472 fibres, as shown in Figure.8-a. To simplify the model, the paper makes the following assumptions:

1 The thickness of the interphase is very small;

2 The initial stiffness of interphase is large enough.

In this case, the effect of the interface on the performance of the single cell can be ignored. In particular, the three-dimensional solid element is replaced by a two-dimensional plane strain unit due to its transverse isotropic concept, which will greatly reduce units number and save computing time.

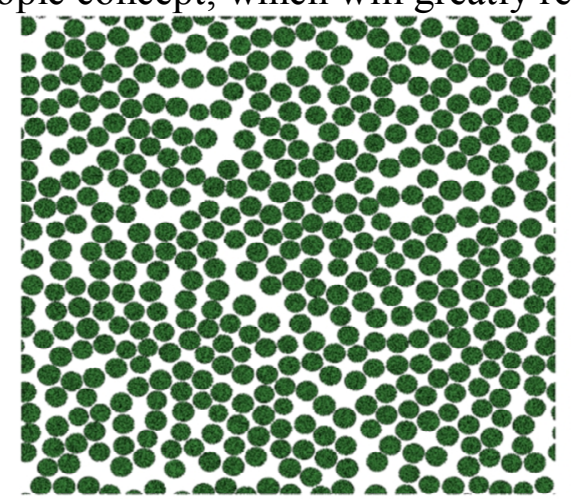

a)Fibres(green part)

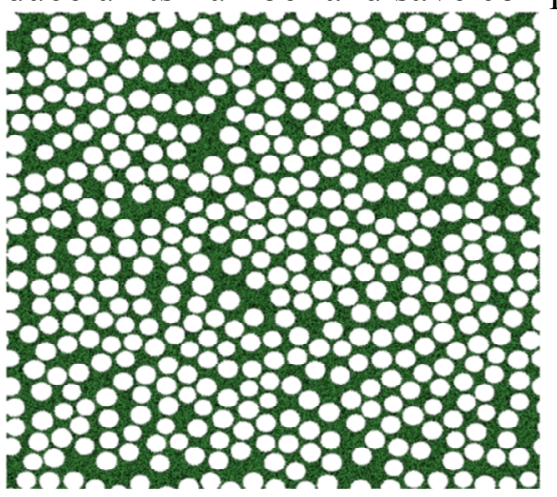

b)Matrix(green part)

Figure. 8 The sketch map of RVE finite element model

For a certain RVE model, it must have the consentaneous deformation with its neighboring cell to satisfy continuity conditions of stress and displacement continuity conditions ${ }^{[21]}$. Xia Z.et.al ${ }^{[22]}$ has proved that the stress of continuous natural conditions can be satisfied when the displacement continuity conditions are met. In the paper, the Python scripting language ABAQUS/CAE is used to achieve random distribution parametric RVE model generation.

\section{Result analysis}

For the T300/BMP-316 macro elastic properties of the fibre are predicted and shown in Table 1.As 
can be seen from the table that all the property prediction results are very close to but smaller than the experimental values. This discrepancy may be the reason that interphase exists in the real material structures but the interphase strength is ignored in the paper. At the same time, in a real manufacture circumstance, porosities and other processing errors will have a certain impact on the properties of composite materials.

Table 1 Predicted elastic properties of T300/BMP-316

\begin{tabular}{ccccc}
\hline & Experimental $^{[23]}$ & $\begin{array}{c}\text { Predicted } \\
\text { results }\end{array}$ & Error $(\%)$ & $\begin{array}{c}\text { Standard } \\
\text { deviations }\end{array}$ \\
\hline$E_{1}(\mathrm{MPa})$ & 128.8 & 124.37 & 3.43 & 0.145 \\
$E_{2}(\mathrm{MPa})$ & 8.94 & 8.14 & 8.95 & 0.164 \\
$G_{12}(\mathrm{MPa})$ & 5.66 & 5.33 & 5.83 & 0.0183 \\
$G_{23}(\mathrm{MPa})$ & 3.03 & 2.87 & 5.28 & 0.0109 \\
$v_{12}$ & 0.328 & 0.297 & 9.45 & 0.0007 \\
$v_{23}$ & 0.477 & 0.434 & 9.01 & 0.0011 \\
\hline
\end{tabular}

\section{Conclusion}

1) The fibre features in the composite microstructure SEM image can be received to reconstruct the composite finite element models accurately by obtaining microscopic distribution state of fibres through image processing methods.

2) The random sequence generation algorithm has been proposed to generate statistically equivalent fibre distributions. Comparison with experiment results and different statistical functions show that the RVE produced by it substantially coincide to meet the requirements of statistical equivalence distributions and is more suitable to simulate real fibre distributions situation than other methods mentioned in the paper.

3) The paper has proved that numerical simulation method on composite materials elastic performance can accurately achieve the macro mechanical composite materials properties compared with the experimental results by means of calculating the micro stress strain field of RVE model under different uniaxial loads with volume homogenization.

\section{References}

[1] Jianqiao Chen. Introduction to mechanics of composite materials[M].Beijing, Science Press, 2006. (in Chinese)

[2] Shan Zhaohui,Gokhale AM..Representative volume element for non-uniform micro-structure [J]. Computational Materials Science,2002;24(3):361-79.

[3] Aghdam M M,Dezhsetan A.Micromechanics based analysis of randomly distributed fibre reinforced composites using simplified unit cell model[J].Composite Structure,2005;71:327 332.

[4] Sun Changjie, Saffari Payman, et al. Finite element analysis of elastic property bounds of a composite with randomly distributed particles[J]. Composite ,Part A,2007;38:80 86.

[5] Hojo M,Mizuno M,Hobbiebrunken T,Adachi T,Tanaka M,Ha SK. Effect of fibre array irregularities on microscopic interfical normal stress states of transversely loaded UD-CFRP from viewpoint of failure initiation [J]. Compos Sci Technol ,2009;69(11-12):1726-34

[6] Vaughan TJ,McCarthy CT.A combined experimental-numerical approach for generating statistically equivalent fibre distributions for high strength laminated composite materials[J]. Compos Sci Technol,2010;70(2):291-7.

[7] Melro AR,Camanho PP,Pinho ST.Generation of random distribution of fibres in long-fibre 
reinforced composites[J]. Compos Sci Technol,2008;68(9):2092-102.

[8] Matsuda T,Ohno N,Tanaka H,Shimizu T. Effects of fibre distribution on elastic-viscoplastic behaviour of long fibre-reinforced laminates [J]. Int. J. Mech. sci. 2003;45(10):1583-98.

[9] Romanowicz M. Progressive failure analysis of unidirectional fibre-reinforced polymers with inhomogeneous inter phase and randomly distributed fibres under transverse tensile loading. Compos Part A - Appl Sci Manuf ,2010;41(12):1829-38.

[10] Lei Yang,Ying Yan,Zhiguo Ran,Yujia Liu.A new method for generating random fibre distribution for fibre reinforced composites[J]. Compos Sci Technol,2013;76:14-20.

[11] Xiaoqiang Wang.Meso-Finite Element Analysis of the Tensile Properties of Composites Based on Cohesive Model [D] . Harbin Engineering University, 2012.(in Chinese)

[12] Zhongyang. Stuhy on Prediction of Mechanical Performance for Unidirectional Composite Considering Interphase [D] . Harbin Institute of Technology, 2010. (in Chinese)

[13] Liju Dong. 图像阈值化技术的综述、分类及评价[J] . Journal of Shenyang University, 2004,16(4 ): 8 11. (in Chinese)

[14] Pan Y, et al. Analysis of 3D random chopped fibre reinforced composites using FEM and random sequential adsorption[J].Compute Mater Sci,2008;43(3):450 461.

[15] Diggle P. Statistical analysis of spatial point patterns. London:Arnold;2003.

[16] Trias D,Costa J,mayugo JA,HurtadoJE. Random models versus periodic models for fibre reinforced composites[J]. Compute Mater Sci,2006;38(2):316-24.

[17] Pyrz R.Quantitative description of the microstructure of composites. part I:morphology of unidirectional composite systems[J]. Compos Sci Technol ,1994;50(2):197-208.

[18] Valentin Romanov,Stepan V. Lomov, Yentl Swolfs, et al.Statistical analysis of real and simulated fibre arrangements in unidirectional composites[J]. Compos Sci Technol,2013;87:126 134.

[19] Yang S,Tewari A,Gokhale AM.Modeling of non-uniform spatial arrangement of fibres in a ceramic matrix composite[J].Acta Mater 1997;45(7):3059-69.

[20] Trias D. Analysis and simulation of transverse random fracture of long fibre reinforced composites[D]. Thesis, University of Girona, Dep.dEnginyeria Mecanical i de la Construccio Industrial ;2005.

[21] Zimin Pan. Research of the Effect of Interface on the Mechanical Properties of Unidirectional Fiber Reinforced Composites. [D]. Harbin Institute of Technology, 2010. (in Chinese)

[22] Xia Z, Zhou C, Yong Q, et al. On selection of repeated unit cell model and application of unified periodic boundary conditions in micro-mechanical analysis of composites [J]. International Journal of Solids and Structures, 2006, 43(2): 266-278.

[23]Danyong Wang. Research on Prediction of Damage Failure and FatigueLife for Composite Bolted Joints [D].Nanjing University of Aeronautics and Astronautics,2006. (in Chinese) 